\title{
GALACTIC 1.8 MEV EMISSION FROM AL-26
}

\author{
N. PRANTZOS \\ Institut d'Astrophysique de Paris \\ 98bis, Bd. Arago, 75014 Paris
}

\section{Introduction}

The detection of the relatively short-lived $\left(\sim 10^{6} \mathrm{yr}\right)$ radioactive ${ }^{26} \mathrm{Al}$ in our Galaxy, more than 12 years ago, convincingly showed that nucleosynthesis is still active today on a large scale. Still, despite considerable efforts in the past decade, the site of this nucleosynthetic activity cannot be identified from theory alone; several potential sites are still on the list, been able to provide the $2-3 \mathrm{M}_{\odot}$ of ${ }^{26} \mathrm{Al}$ per Myr, implied by the detected $1.8 \mathrm{MeV}$ flux of $\sim 410^{-4} \mathrm{~cm}^{-2} \mathrm{~s}^{-1}$ from the galactic plane (see [7] for a review).

The recent observations of the Compton Gamma-Ray Observatory allowed for the first time to establish a map of the distribution of ${ }^{26} \mathrm{Al}$ in the Galaxy [2], suggesting that massive stars are its most probable sources. On the other hand, the GRIS instrument detected an unexpectedly broad line [4], with potentially important implications for our understanding of the fate of nucleosynthesis ejecta [1]. Future observations with the INTEGRAL satellite are expected to clarify this point and to reveal details of the $1.8 \mathrm{MeV}$ map, corresponding to major sites of galactic nucleosynthesis.

\section{Production sites of Al-26}

Several astrophysical sites may produce important amounts of ${ }^{26} \mathrm{Al}$, by hydrostatic or explosive nucleosynthesis [7].

- Wolf-Rayet (WR) stars, with initial mass $M>30 \mathrm{M}_{\odot}$ (for solar metallicity) synthesize ${ }^{26} \mathrm{Al}$ in their H-burning cores and eject it in the ISM by their strong stellar winds. The most recent models of WR stars [3], with improved physical ingredients (mass loss and nuclear reaction rates, opacities) account better than previous ones for observed WR properties (luminosities, composition, statistics) and show that galactic WR stars could produce 
up to $\sim 1 \mathrm{M}_{\odot}$ of ${ }^{26} \mathrm{Al}$ in the past Myr; uncertainties in stellar models may reduce this figure by a factor $\sim 2-3$.

- Models of 10-30 $\mathrm{M}_{\odot}$ stars (with no mass loss) show that they synthesize ${ }^{26} \mathrm{Al}$ hydrostatically in their Ne layers and (to a smaller extent) explosively in their C-Ne layers, during the SNII explosion; in the latter case, neutrino induced nucleosynthesis may also produce additional ${ }^{26} \mathrm{Al}$. The amount of hydrostatically produced ${ }^{26} \mathrm{Al}$ is subject to important uncertainties, related to the treatment of convection in the corresponding stellar layers, and so is the result of $\nu$-nucleosynthesis (due to its sensitivity to the poorly known $\nu$ spectra). Massive stars may produce up to $2 \mathrm{M}_{\odot}$ of ${ }^{26} \mathrm{Al}$ per Myr [8], but 3-5 times smaller amounts cannot be excluded.

-Intermediate mass stars $\left(\sim 4-9 \mathrm{M}_{\odot}\right)$ can produce important amounts of ${ }^{26} \mathrm{Al}$ during their AGB phase, by hydrostatic H-burning in the bottom of their convective envelopes, if the temperature in that zone is $\mathrm{T}>710^{7} \mathrm{~K}$. Although some Hot Bottom Burning may indeed take place in those stars, the amount of ${ }^{26} \mathrm{Al}$ produce is highly uncertain, due to our current inability to evaluate the depth of the convective envelope.

- Novae may also synthesize interesting amounts of ${ }^{26} \mathrm{Al}$ by explosive $\mathrm{H}$ burning, but the uncertainties involved in the current calculations (related mainly to the treatment of time-dependent convection, even in hydrodynamic models) do not allow any definite predictions.

In summary, several nucleosynthesis sites may be able to provide $\sim 2$ $\mathrm{M}_{\odot}$ of ${ }^{26} \mathrm{Al}$ per $\mathrm{Myr}$, with theoretical uncertainties been much larger in the case of of novae and AGB stars than in the case of SNII or WR stars.

\section{Diffuse galactic $1.8 \mathrm{MeV}$ emission from ${ }^{26} \mathrm{Al}$}

In view of the difficulty to decide on purely theoretical grounds on the most plausible source of galactic ${ }^{26} \mathrm{Al}$, it was suggested that a clear signature could be provided by the spatial profile of the $1.8 \mathrm{MeV}$ emission in the galactic plane: an asymmetric profile with prominent features in the tangent directions to the spiral arms, would favour massive stars (WR, SNII), while a smooth profile would favour an old population (low mass AGB stars and novae).

The COMPTEL instrument aboard the Compton GRO performed the first mapping of the $1.8 \mathrm{MeV}$ line in the early 90ies [2]. The results of the 5-year survey (1991-1996) appear on Fig. 1. The emission profile is clearly assymetric and, besides the inner Galaxy, several prominent features appear, three of which (at galactic longitude $1 \sim 32^{\circ},-50^{\circ}$ and $-74^{\circ}$ ) can be tentatively identified with spiral arms. This is a strong argument for a massive star origin of the galactic ${ }^{26} \mathrm{Al}$ although, in principle, massive AGB stars with $\mathrm{M}>5 \mathrm{M}_{\odot}$ or $0 \mathrm{NeMg}$ rich novae from progenitors of some- 

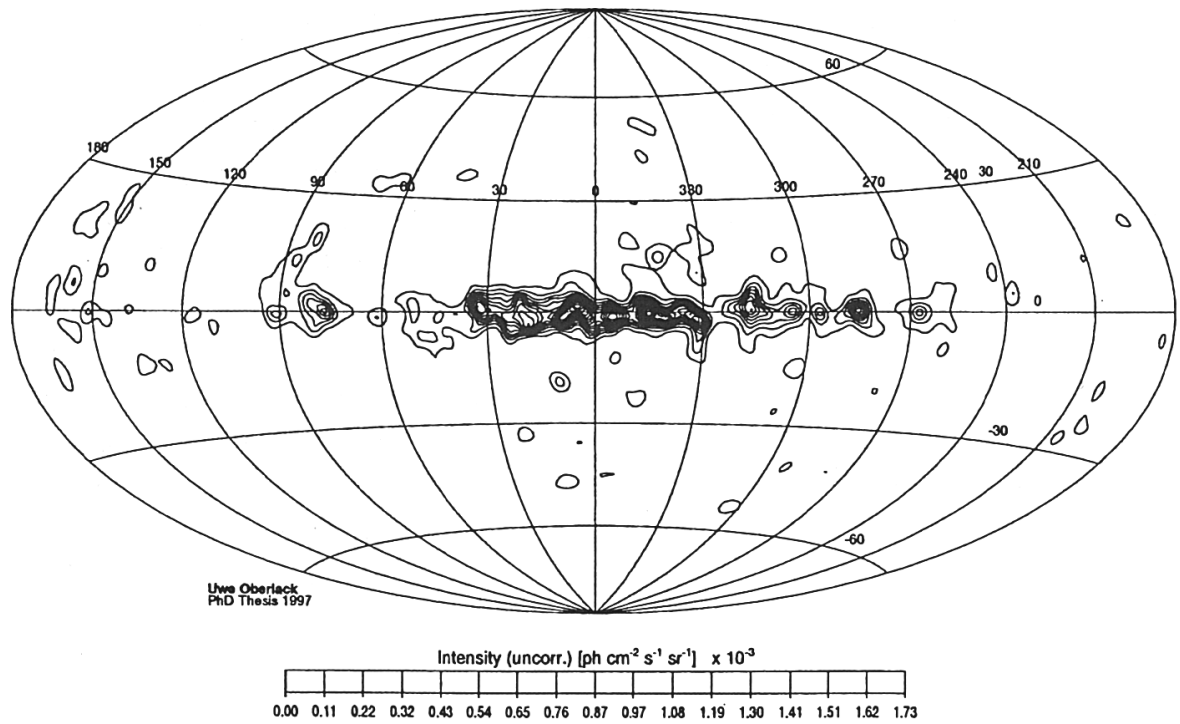

Figure 1. Sky-map of $1.8 \mathrm{MeV}$ emission, from the 5-year survey of COMPTEL (1991-1996). The emission is concentrated in the galactic plane, and mostly in the inner Galaxy $\left(-30^{\circ}\right.$ to $+30^{\circ}$ of galactic longitude), but also in several hot-spots; some of them (at $1 \sim 32^{\circ},-50^{\circ},-74^{\circ}$ ) can be tentatively identified with spiral arms, while others with more localised nearby sources like Vela $\left(\sim-90^{\circ}\right)$ or Cygnus $\left(\sim 80^{\circ}\right)$.

The overall picture (emission assymetric w.r.t. the Galactic Center, and several hot-spots identified with spiral arms), points to massive stars at the origine of Al-26. (Courtesy: R. Diehl and the COMPTEL team).

what higher mass cannot be excluded. A composite picture, with a smooth background from low mass sites and a superimposed massive stellar population concentrated in the spiral arms cannot be excluded either, but the overall picture loses in simplicity.

Two of the remaining hotspots $\left(1 \sim 80^{\circ},-90^{\circ}\right)$ are certainly not related to spiral arms. The former can be identified with the Cygnus region, where the activity of several tens of SN in the past Myr can account for the observed emissivity. The latter coincides with the Vela region and originates probably from the Vela remnant (age: $\sim 11000 \mathrm{yr}$ ). This detection, the first of a point source of ${ }^{26} \mathrm{Al}$, would allow to calibrate the yield of individual SNII, once the still uncertain distance to Vela $(200-400 \mathrm{pc})$ is more precisely evaluated.

In a recent balloon flight, the GRIS experiment (with a large field of view, of $\sim 100^{\circ}$, but with a unique spectroscopic capability in this energy range, due to its $\mathrm{Ge}$ detector) detected the $1.8 \mathrm{MeV}$ emission from the Galactic Center direction at the $\sim 7 \sigma$ level. The line profile is surprisingly broad, with an intrinsic FWHM W=5.4 $1.4 \mathrm{keV}$ [4]. This corrsponds to a Doppler velocity of $540 \pm 140(\mathrm{~W} / 5.4) \mathrm{km} / \mathrm{s}$ for a spherical expansion 
or to an effective temperature $k T \sim 39 \pm 20 \mathrm{keV}\left(T \sim 4.5 \pm 2.310^{8} \mathrm{~K}\right)$ for a thermal origin. Indeed, the line was expected to be rather narrow, its width reflecting only galactic differential rotation or random motions of the ISM, in which ${ }^{26} \mathrm{Al}$ was supposed to be at rest (thermalised in a short time $<<1$ Myr after its ejection). But galactic rotation alone can account only for a FWHM $\sim 1.7 \mathrm{keV}$ only, i.e. 3-4 times smaller than observed.

If confirmed, this result will certainly have important implications for our understanding of the fate of nucleosynthesis ejecta. Some of these implications have been scetched in [1]; the most interesting concerns probably the possibility for the ejected metals (from either WR, SNII or novae, but not from AGB stars, their winds been very slow) to condense in grains and travel undeccelerated for timescales of $\sim 1$ Myr. More refined calculations and a better understanding of the grain properties are necessary to find whether this idea is realistic or not.

\section{Conclusions}

A major step in our understanding of the nucleosynthesis of ${ }^{26} \mathrm{Al}$ in our Galaxy was achieved in the 90ies, with the first mapping of the galactic $1.8 \mathrm{MeV}$ emission by COMPTEL. The assymetric profile of the emission and the (quite probable) identification of several prominent features with spiral arms clearly favour massive stars (WR and/or SNII) as the sources of ${ }^{26} \mathrm{Al}$ (although a contribution from lower mass objects, like AGB stars and novae, cannot be excluded).

Future observations with the INTEGRAL satellite, to be launched in 2001-2002, are expected to reveal details of that map (corresponding to major sites of galactic nucleosynthesis), to help establishing the poorly known spiral pattern in the inner Galaxy, to allow calibration of the ${ }^{26} \mathrm{Al}$ yields of individual sites (through confirmation of the Vela emission and detection of other point sources) and to improve our understanding of the fate of nucleosynthesis ejecta (in case the reported width of the $1.8 \mathrm{MeV}$ line is confirmed).

\section{References}

1. Chen W. et al., 1997, in The Transparent Universe, Eds. T. Courvoisier \& Ph. Durouchoux (ESA), in press

2. Diehl R. et al., 1995, A\&A, 298, 445

3. Meynet G. et al., 1997, A\&A, 320, 460

4. Naya J. et al., 1996, Nature, 384, 44

5. Oberlack et al., 1994, ApJS, 92, 433

6. Prantzos N., 1993, ApJ, 405, L55

7. Prantzos N. \& Diehl R., 1996, Phys. Rep., 267, 1

8. Timmes F. et al., 1995, ApJ, 449, 204 